\title{
Primary cauda equina lymphoma diagnosed by nerve biopsy: A case report and literature review
}

\author{
KAYO SUZUKI $^{1}$, TAKETOSHI YASUDA ${ }^{1}$, TOSHIHITO HIRAIWA ${ }^{1}$, MASAHIKO KANAMORI $^{2}$, \\ TOMOATSU KIMURA $^{1}$ and YOSHIHARU KAWAGUCHI ${ }^{1}$
}

Departments of ${ }^{1}$ Orthopaedic Surgery and ${ }^{2}$ Human Science 1, Faculty of Medicine, University of Toyama, Toyama, Toyama 930-0194, Japan

Received December 29, 2017; Accepted April 26, 2018

DOI: $10.3892 / \mathrm{ol} .2018 .8629$

\begin{abstract}
Primary cauda equina lymphoma (CEL) is a rare malignant tumor among various neoplasms that affects the cauda equina nerve roots. The present case report described the case of a 65-year-old man who presented with cauda equina syndrome with progressive motor palsy in the legs and gait disturbance over the last 5 months. Magnetic resonance (MR) images showed enlargement of the cauda equina occupying the dural sac from the L1-S1 level with isointensity to the spinal cord signal on both T1- and T2-weighted imaging. Enhancement of the cauda equina was seen on contrast MR images. On F-18 2-fluoro-2-deoxy-glucose positron emission tomography examination, diffuse accumulation of 2-fluoro-2-deoxy-glucose was observed in the cauda equina with a maximum standardized uptake value of 4.9. Based on elevation of soluble interleukin 2 receptor in cerebrospinal fluid and a biopsy of the enlarging cauda equina, a diagnosis of CEL of the diffuse large B-cell type was made. The present case report provided a detailed case discussion and a review of the available literature on this rare entity, focusing on clinical characteristics and imaging of primary CEL.
\end{abstract}

Correspondence to: Dr Kayo Suzuki, Department of Orthopaedic Surgery, Faculty of Medicine, University of Toyama, 2630 Sugitani, Toyama, Toyama 930-0194, Japan

E-mail: suzukayo@med.u-toyama.ac.jp

Abbreviations: NL, neurolymphomatosis; CEL, cauda equina lymphoma; FDG-PET/CT, 2-fluoro-2-deoxy-glucose positron emission tomography/computed tomography; WBC, white blood cell; sIL-2R, soluble interleukin 2 receptor; MR, magnetic resonance; SUV, standardized uptake value; CSF, cerebrospinal fluid; CD, cluster of differentiation; BCL2, B-cell lymphoma 2; MUM-1, multiple myeloma oncogene 1; DLBCL, diffuse large B-cell lymphoma; PCNSL, primary central nervous system lymphoma

Key words: cauda equina lymphoma, nerve biopsy, MR imaging, FDG-PET/CT, sIL-2R

\section{Introduction}

Malignant lymphoma arising in the cauda equina is included in the entity of neurolymphomatosis (NL), which is characterized by the infiltration of malignant lymphoma cells into peripheral nerves, nerve roots, plexuses, or cranial nerves $(1,2)$. NL is classified as primary and secondary. Primary NL is defined as NL that is the first manifestation of the hematologic malignancy, and secondary NL is defined as NL that is the site of relapse or progression of a previously diagnosed lymphoma or leukemia (1). NL is a very rare malignant lymphoma, comprising only $0.2 \%$ of non-Hodgkin's lymphoma cases (3). Furthermore, the frequency of primary NL is approximately $20 \%$ of all NL cases and is less frequent than secondary NL (4). Therefore, primary NL is a rare entity. Primary cauda equina lymphoma (CEL) as primary NL is an extremity rare condition in which lymphoma cells primarily infiltrate the cauda equina. In previous reports, only 22 cases of primary CEL have been reported. Because primary CEL is an uncommon condition, detailed information about clinical features and image findings has not been reported yet. Furthermore, the clinical symptoms of CEL vary and may include mild to severe muscle weakness or numbness of lower limbs, and bladder and bowel dysfunction. These symptoms resemble lumbar spinal stenosis, and thus, diagnosis of CEL may take time. As a result, appropriate treatment may be delayed.

We encountered a case of primary CEL that was diagnosed following F-18 2-fluoro-2-deoxy-glucose positron emission tomography (FDG-PET) and cauda equina biopsy. In this report, we describe an additional case of primary CEL and review the literature with emphasis on clinical characteristics and imaging features of primary CEL.

\section{Case report}

A 65-year-old man presented with gait disturbance due to motor palsy in the bilateral lower extremities over the last 5 months. He also had severe numbness in his left sole. The severity of the symptoms gradually increased, and he was admitted to our hospital in a wheel chair. He had a history of L3-4 laminectomy with a diagnosis of lumbar spinal stenosis at another hospital 1.5 years ago. He also had a urinary stent that had been inserted in another hospital 2 years ago due to a 
diagnosis of benign bladder hypertrophy. Neurological examination on admission showed cauda equina syndrome below the L2 level. Testing of the motor function of his lower extremities, including the iliopsoas, quadriceps femoris, tibialis anterior, and gastrocnemius muscles, showed marked palsy with a score of 3-4 following evaluation of manual muscle testing. Deep tendon reflexes of the bilateral lower extremities were diminished. Sensory disturbance was also found in the bilateral lower extremities. A complete blood count showed white blood cell (WBC) count; 46.9 $\times 10^{2} / \mu 1$, hemoglobin; $13.1 \mathrm{~g} / \mathrm{dl}$, platelets; $21.7 \times 10^{4} / \mu 1$, and differential WBC count with $59 \%$ neutrophils, $36 \%$ lymphocytes, and $3 \%$ monocytes. No lymphoma cells were found in the peripheral blood. Laboratory data showed that his lactate dehydrogenase was within normal limits (149 U/1), and his C-reactive protein was slightly elevated $(0.41 \mathrm{mg} / \mathrm{dl})$. Soluble interleukin 2 receptor (sIL-2R) was slightly increased (514 U/ml).

Magnetic resonance (MR) images of the lumbar spine demonstrated swollen cauda equina occupying the dural sac from the L1-S1 level that was isointense on T1-weighted MR images and hypointense on T2-weighted MR images compared to the spinal cord (Fig. 1A). Gadolinium-enhanced T1-weighted MR images revealed swelling of the cauda equina nerve roots with diffuse enhancement (Fig. 1B and C). FDG-PET showed diffuse accumulation of FDG in the cauda equina with a maximum standardized uptake value (SUV) of 4.9 (Fig. 2). Examination of cerebrospinal fluid (CSF) following a lumbar spinal tap showed that the cell count was $443 / \mu 1$, protein was $2470 \mathrm{mg} / \mathrm{dl}$, and glucose was less than $2 \mathrm{mg} / \mathrm{dl}$. sIL-2R in CSF was remarkably increased to $2,033 \mathrm{U} / \mathrm{ml}$, and cytology of the CSF was defined as stage IV. To obtain more detailed pathological information, we performed a cauda equina biopsy during motor evoked potential monitoring. After L4-5 partial laminectomy and incision of the dural sac, the cauda equina was observed as extremely swollen with grayish tumors present. The tumors infiltrated extensively into the cauda equina and adhered strongly to the nerve root. We performed the biopsy by cutting one of the cauda equina. The patient did not have any further motor deficits, severe leg pain, or numbness after surgery. Pathological examination was performed (Fig. 3). The pathological examination revealed that atypical cells with irregular large nuclei and little cytoplasm had infiltrated into the nerve (Fig. 3A and B). Immunohistochemistry revealed that the atypical large cells were positive for cluster of differentiation (CD)20, B-cell lymphoma 2 (BCL2), BCL6, multiple myeloma oncogene 1 (MUM-1), and negative for CD3, CD5 and CD10 (Fig. 3C-E, G, H). The nerve region which was showed by 'single asterisk' in the Fig. 3A was positive by S-100 staining (Fig. 3F). Thus, we diagnosed the pathology as diffuse large B-cell lymphoma (DLBCL), non-germinal center type that originated in the cauda equina.

He was treated with intravenous chemotherapy using high-dose cytarabine plus high-dose methotrexate for four cycles in 4 months. The severe adverse events of this regimen were Grade 3-4 anemia and neutropenia, which required blood transfusion and administration of granulocyte-colony stimulating factor. The chemotherapy was effective. After chemotherapy was over, we recommended radiotherapy to the patient, but because the patient strongly refused, we followed up with him without radiotherapy. His motor function was restored following rehabilitation to correct the muscle weakness in the lower limbs, and he was able to walk without any assistance 6 months after induction of chemotherapy. MR images of the lumbar spine did not show any enhancement in the cauda equina at 3 months after completion of chemotherapy (Fig. 4). He has maintained complete remission for more than 6 years after the initial diagnosis of CEL.

This study was approved by the Ethics Committee of the Toyama University Hospital (Toyama, Japan), Database of Musculoskeletal Disease (no. 21-22) and Database of Musculoskeletal Tumor (no. 24-40). The patient gave his written consent for this report.

\section{Discussion}

Lymphoma cells rarely infiltrate nervous system tissues. When malignant lymphoma occurs in the central nervous system, it is called primary central nervous system lymphoma (PCNSL), and PCNSL accounts for 4-6\% of all malignant lymphomas (5). When malignant lymphoma infiltrates the peripheral nervous system, it is called NL (1). Among primary NLs, invasion of the cauda equina by lymphoma cells is extremely rare. Twenty-three cases of primary CEL, including our case, have been reported so far (2,6-25). We reviewed these 23 cases regarding the clinical characteristics and imaging of primary CEL and summarized these data in Table I. Among the reported cases of primary CEL, the youngest patient was 11 years old (18), but the median age of CEL patients is 55 years. The male-to-female ratio is 1.3:1, which is similar to that of PCNSL (5). PCNSL has an increased incidence in immunocompromised hosts, such as patients with acquired immunodeficiency syndrome, post-organ transplantation, and congenital immunodeficiencies (26). In this series, immunocompromised hosts included only two cases $(8.7 \%)$, one with acquired immunodeficiency syndrome and one with Epstein-Barr virus infection $(8,11)$. The duration from onset of symptoms to diagnosis was 6 months on average. The shortest duration was 3 days (18), but in some cases, 3 years elapsed before diagnosis of primary CEL $(13,14)$. Symptom onset varies from acute to subacute progression. Major symptoms include lower back pain, muscle weakness, and numbness of lower limbs, with or without mild to severe urinary dysfunction. In some cases, the symptoms progress rapidly (18-20). Neurological examination showed cauda equina syndrome in 17 cases including our case (74\%) $(2,6-10,12,13,15,17-21,23,24)$, radiculopathy in three cases $(13 \%)(16,22)$, and other symptoms (polyradiculoneuropathy, irritation, and facial numbness) in three cases $(13 \%)(11,14,25)$. Because CEL involves some cauda equina roots, many patients with CEL present with cauda equina syndrome.

sIL-2R is expressed after B cell activation and is a strong independent prognostic factor even for long-term observation during treatment for DLBCL (27). The serum level of sIL-2R is not a specific and highly sensitive marker of PCNSL, but serial evaluation of sIL-2R may be useful for monitoring therapeutic effectiveness (28). Among the previous reports of primary CEL, only a case presented by Tajima et al showed elevation of sIL-2R in serum and CSF (14). Our present case also had elevated levels of sIL-2R slightly in serum and markedly in CSF. Because sIL-2R in CSF was markedly 

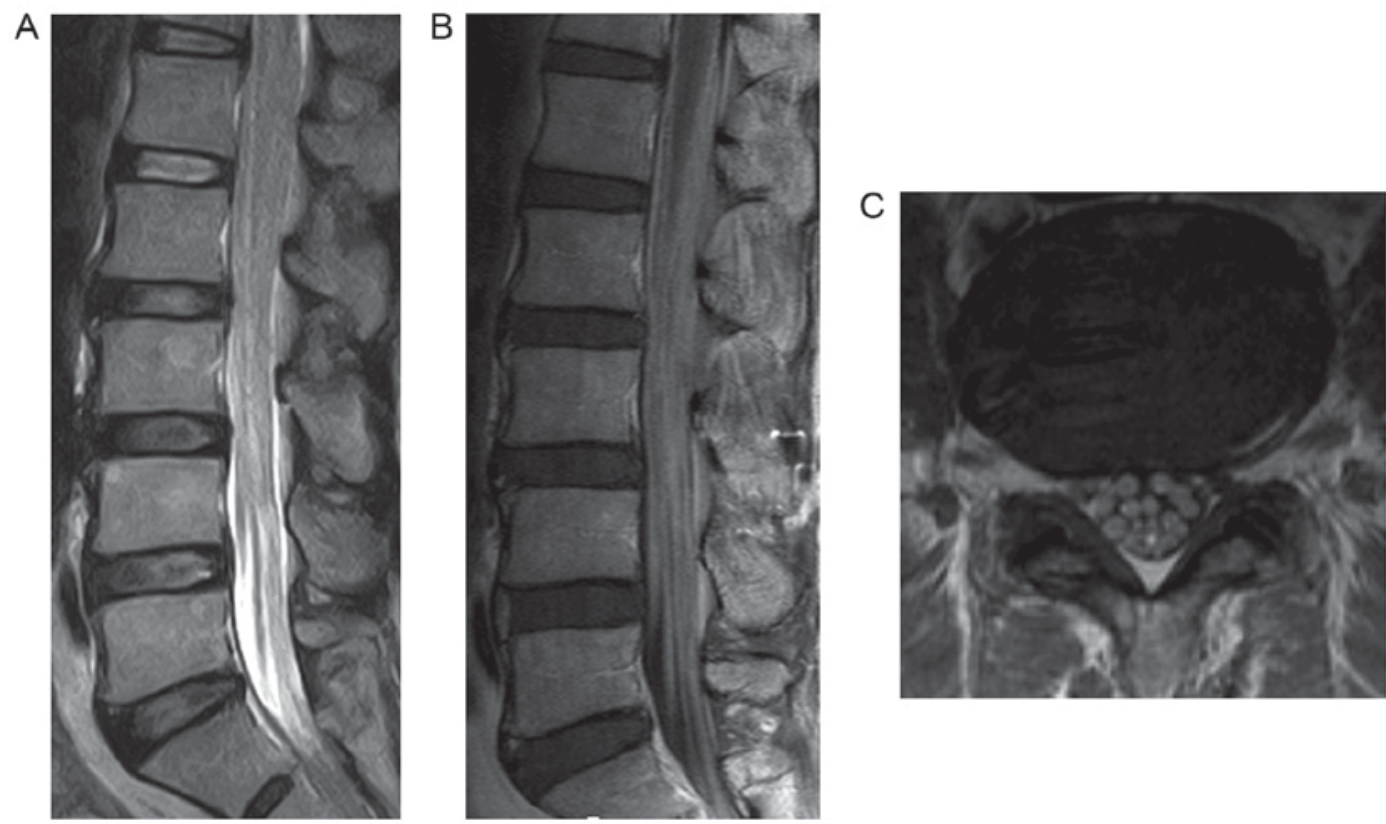

Figure 1. Magnetic resonance images of a cauda equina lesion. (A) The cauda equina showed low signal intensity compared to the conus on a T2-weighted image. (B) On a sagittal enhanced T1-weighted image, the cauda equina roots showed diffuse enhancement from the level of the L1 vertebra to S1. (C) At the level of the L4/5 disc, each cauda equina root was obviously enlarged.

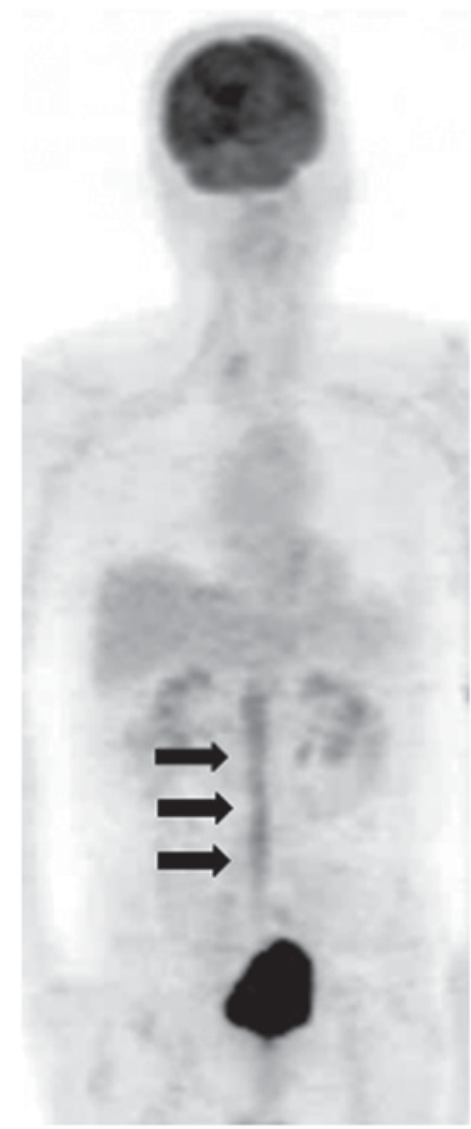

Figure 2. FDG-PET. At the level from the conus to the cauda equina (arrows), diffuse accumulation of FDG was observed with a maximum SUV of 4.9 . FDG-PET, F-18 2-fluoro-2-deoxy-glucose positron emission tomography; SUV, standardized uptake value.

elevated similar to the report by Tajima et al, the sIL-2R level, especially in CSF, could be quite useful for diagnosis of
CEL (14). However, only a few reports of the level of sIL-2R in CSF are available, and the utility of measuring sIL-2R in CSF and its cut-off value are not known in CEL patients. Examination of CSF is helpful for the diagnosis of meningitis, malignant tumors of the spinal cord including CEL, and meningeal dissemination. In these diseases, CSF findings generally show increased leukocytes, elevated protein levels, and decreased glucose. In previously reported cases, an increase in leukocytes was observed in $82.4 \%$ of patients, elevation of protein level in $88.2 \%$, and a decline in glucose in $41.2 \%$ (Table I). However, distinguishing spinal cord tumors from infections with these CSF findings is difficult. Nishida et al reported that cytology and immunophenotyping of CSF are effective diagnostic tools for CEL (20). In addition to general CSF examinations such as the cell count and protein and glucose levels, if atypical cells are observed with CSF cytology, immunophenotyping analysis could improve the accuracy of diagnosis of CEL. In this review of reported articles, only three cases were diagnosed with cytology and CD20 immunotyping of CSF $(11,20,25)$. Furthermore, because a lumbar puncture for obtaining CSF is quickly performed, Nishida et al reported that cytology of CSF is useful for monitoring molecular disease recurrence (20). However, the majority of reported cases were diagnosed with histopathological examination of tumors obtained during surgery or biopsy of the tumor that affected the cauda equina nerve. Broen et al recommended an early biopsy of the nerve root for a definitive diagnosis (22). The possibility of irreversible nerve damage due to nerve root biopsy is a concern, but the effectiveness of obtaining a definitive diagnosis is thought to exceed that risk. In particularly, biopsy of the infiltrated nerve is also recommended for diagnosis of chronic inflammatory demyelinating polyradiculopathy (29). In our present case, we were able to obtain a definitive diagnosis following a biopsy of the cauda equina. The major histopathological type 

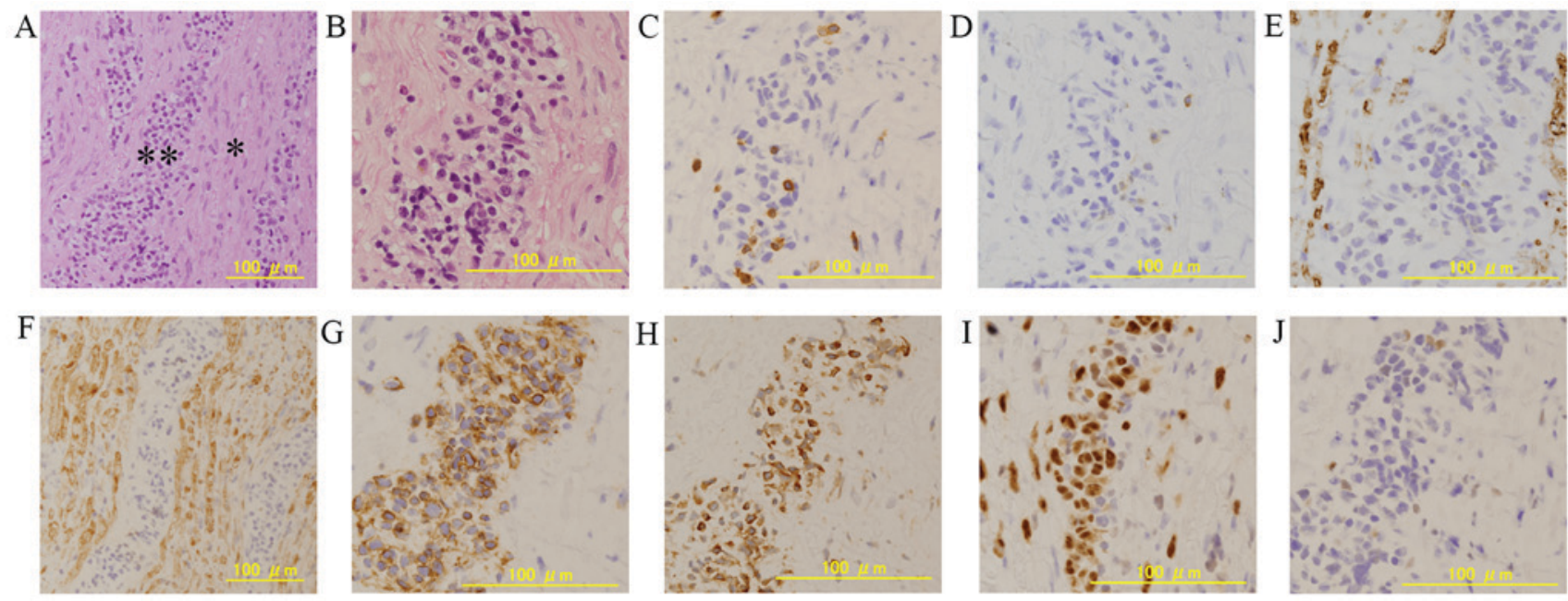

Figure 3. Histopathological examination and immunohistochemistry of the cauda equina following a biopsy. (A) Hematoxylin and eosin staining revealed lymphoma cells (") infiltrated into the nerve $\left(^{* *}\right)$ (magnification, x100). (B) Lymphoma cells presented a large nuclei and little cytoplasm with a high magnification view (magnification, x200). Immunohistochemistry showed that the atypical large lymphoma cells were negative for (C), CD3, (D) CD5, and (E) CD10 (magnification, x200). (F) The nerve region infiltrated by lymphoma cells in figure A was positive by S-100 staining (magnification, $x 100)$. Immunostaining for (G) CD20, (H) BCL2, and (I) MUM-1 were diffusely strong positive for the atypical large lymphoma cells (magnification, x200). Immunostaining for (J) BCL6 was weakly positive for the atypical large tumor cells (magnification, x200). CD, cluster of differentiation; BCL2, B-cell lymphoma 2; MUM-1, multiple myeloma oncogene 1 .

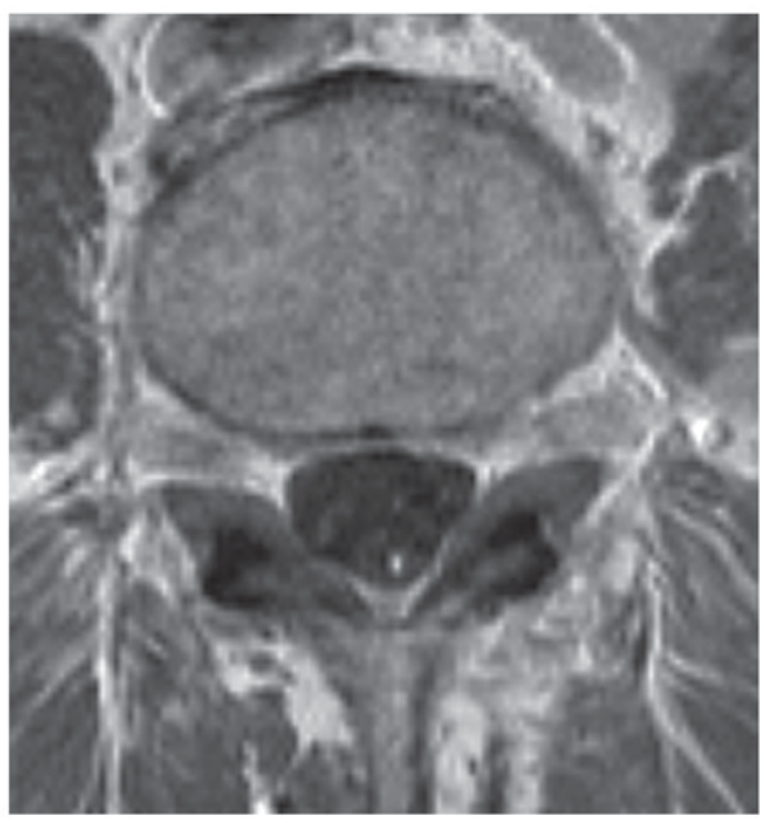

Figure 4. Gadolinium-enhanced T1-weighted magnetic resonance images at 3 months after the completion of chemotherapy. On an axial image at the level of the $\mathrm{L} 4 / 5$ disc, the cauda equina roots showed disappearance of enhancement.

of CEL was the diffuse large B-cell type, which makes up approximately $82.6 \%$ of reported cases of CEL. The T-cell type and natural killer/T cell type are rare subtypes $(10,15)$.

The imaging findings of these 23 cases of primary CEL are summarized in Table II. MR images are useful for assessing the location of lesions and morphology of the cauda equina. Multiple levels of the spine are involved, from T11 to S1. In more than $60 \%$ of the 23 reported cases, CEL extended to the L1 to L4 level (Fig. 5). The most common MR finding of CEL is swelling or enlargement of the cauda equina root $(2,8-25)$. Enlarging nerve roots in CEL exhibit mostly isointensity or low intensity relative to the signal of the spinal cord on T1-weighted images $(2,10,15,20,21,25)$, and high signal intensity may be observed in rare cases $(8,9)$. On T2-weighted images, enlarging nerve roots of CEL show low or isointensity relative to the spinal cord in $78 \%$ of cases $(2,13-15,18,21-23,25)$, and approximately $20 \%$ of cases show slight hyperintensity $(17,19,20)$. In all cases in which contrast MR imaging was performed, the enlarged cauda equina exhibited a contrast effect $(2,9-16,19-25)$. The features of MR images of CEL are enlargement of the cauda equina with iso- or low intensity relative to the spinal cord signal on both T1- and T2-weighted images and the presence of enhancement of the cauda equina on contrast MR images. The findings of MR images in our case also showed enlargement of the cauda equina with isointensity on T1-weighted images, low intensity on T2-weighted images, and enhancement in contrast images, which were consistent with the representative findings of CEL. Enlargement of the cauda equina on MR imaging is observed not only in CEL, but also in chronic inflammatory demyelinating polyradiculopathy, neurofibromatosis, malignant peripheral nerve sheath tumors, and metastatic cauda equina carcinoma tumors (25). Distinguishing between the above-mentioned diseases and CEL with only MR image findings is difficult. Therefore, elevation of protein and SIL-2R levels in the CSF, CSF cytology, and CD20 immunophenotyping of CSF are considered useful for adjunctive diagnosis of CEL. Ultimately, a nerve root biopsy of the cauda equina can lead to a definitive diagnosis of lymphoma (22).

FDG-PET/CT examination of CEL was performed in eight cases including our case among those reported after $2008(2,16,20-22,24,25)$. The findings of FDG-PET/CT show increasing FDG accumulation in tumor lesions in seven cases $(87.5 \%)$. However, the maximum SUV was reported only for the two cases described by Wang et al (25) and our case; the maximum SUV were 9.6 and 4.9, respectively. These values are very low compared with the median SUV max of 21 (range 8.2-47.1) of general DLBCL (30). Because 


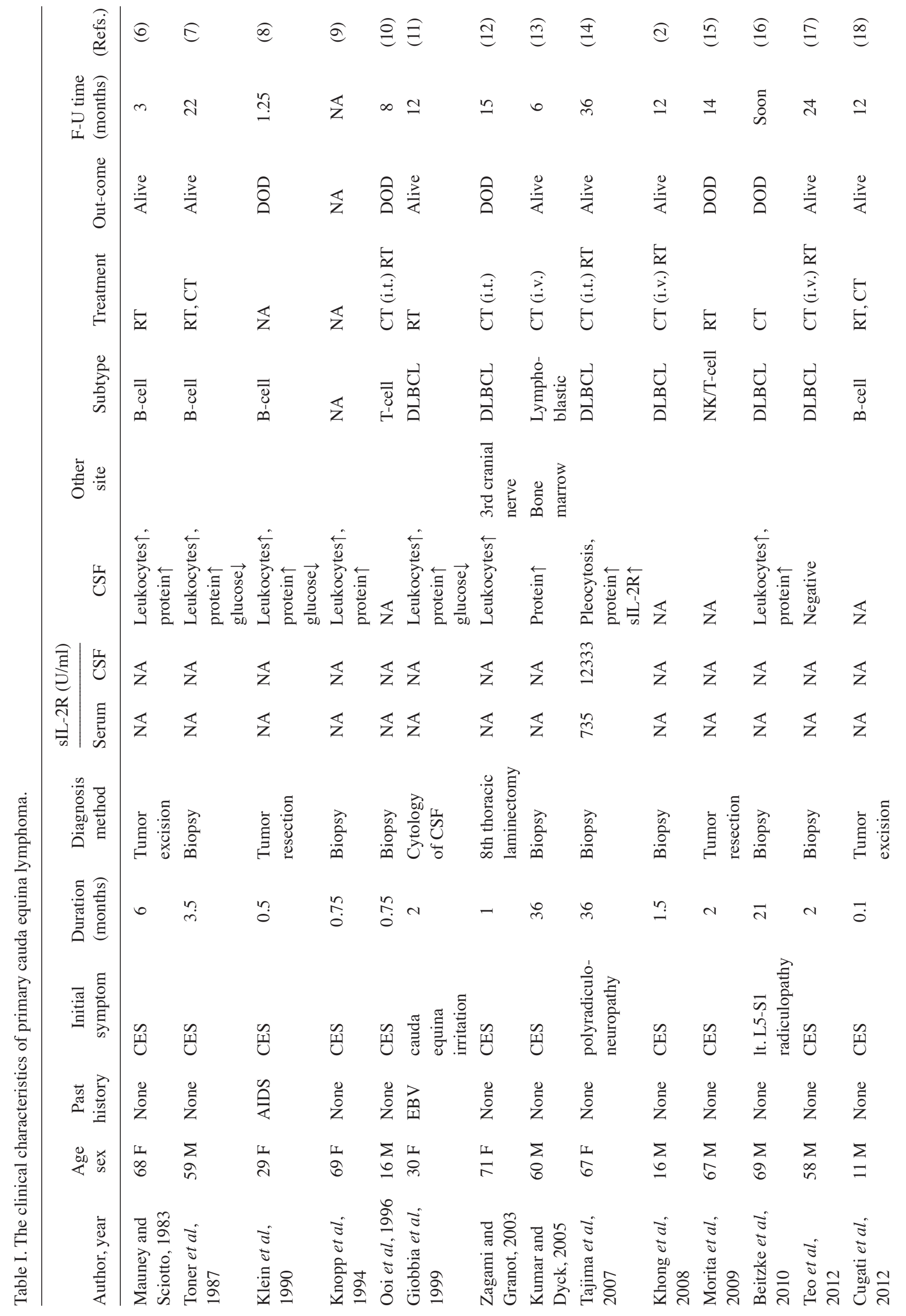




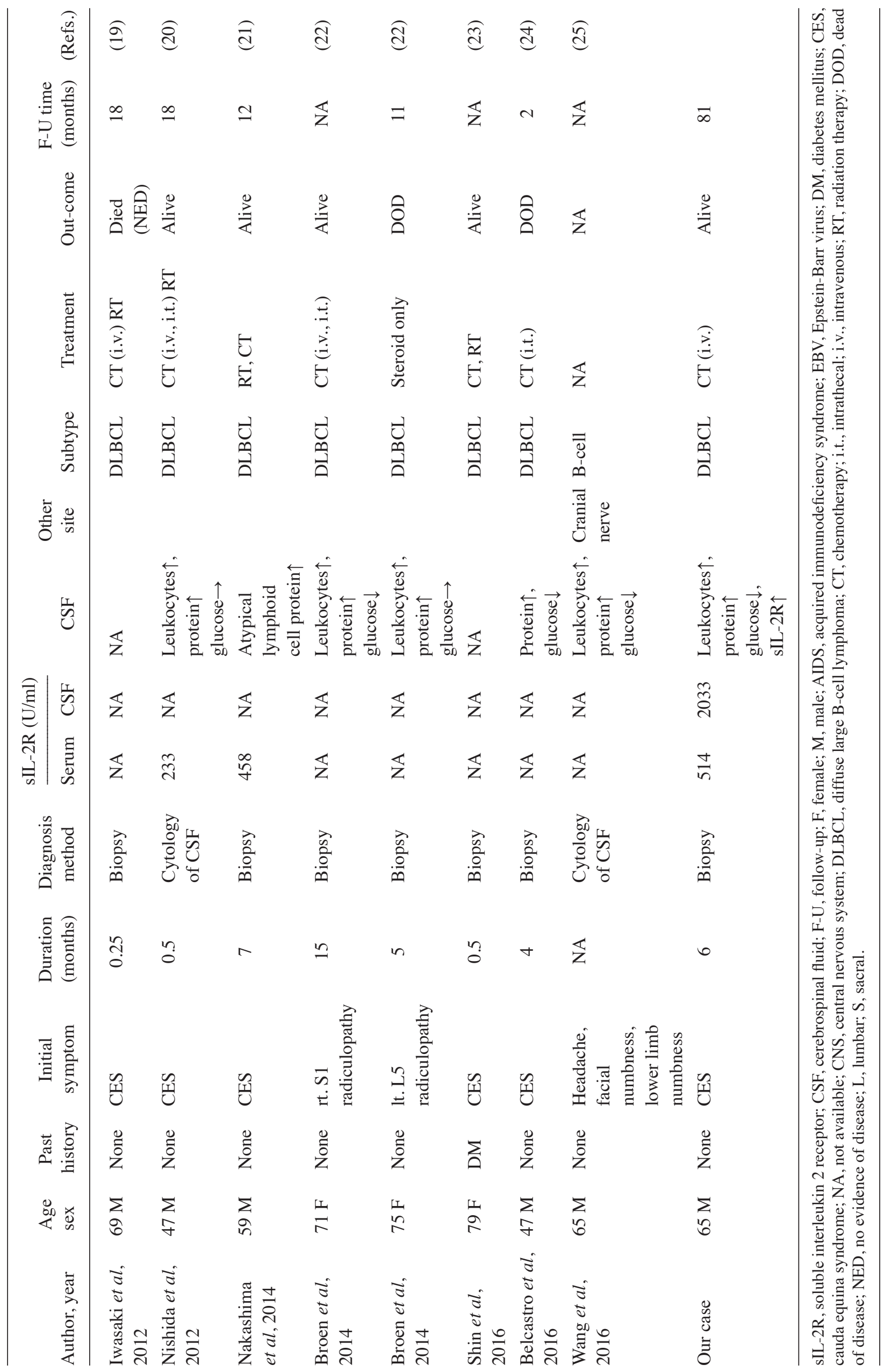


Table II. Imaging findings of primary cauda equina lymphoma.

\begin{tabular}{|c|c|c|c|c|c|c|c|c|}
\hline \multirow[b]{2}{*}{ Author, year } & \multicolumn{5}{|c|}{ MR imaging } & \multicolumn{2}{|l|}{$\mathrm{PET} / \mathrm{CT}$} & \multirow[b]{2}{*}{ (Refs.) } \\
\hline & Level & T1WI & T2WI & Enhancement & $\begin{array}{l}\text { Swelling of } \\
\text { cauda } \\
\text { equina }\end{array}$ & $\begin{array}{c}\text { FDG } \\
\text { accumulation }\end{array}$ & $\begin{array}{l}\text { SUV } \\
\max \end{array}$ & \\
\hline Mauney and Sciotto, 1983 & NA & NA & NA & NA & NA & None & & (6) \\
\hline Toner et al, 1987 & NA & NA & NA & NA & NA & None & & (7) \\
\hline Klein et al, 1990 & L1-L2 & Focally high & NA & NA & + & None & & (8) \\
\hline Knopp et al, 1994 & L1-L3 & $\begin{array}{l}\text { Heterogeneously } \\
\text { high }\end{array}$ & NA & + & + & None & & (9) \\
\hline Ooi et al, 1996 & L2-L4 & Isointense & NA & + & + & None & & $(10)$ \\
\hline Giobbia et al, 1999 & L5-S1 & NA & NA & + & + & None & & $(11)$ \\
\hline Zagami and Granot, 2003 & $\begin{array}{l}\text { Below } \\
\text { cornus }\end{array}$ & NA & NA & + & + & None & & (12) \\
\hline Kumar and Dyck, 2005 & L2-S1 & NA & Low & + & + & None & & (13) \\
\hline Tajima et al, 2007 & T12-L3 & NA & Low & + & + & None & & (14) \\
\hline Khong et al, 2008 & T12-L3 & Low & Low & + & + & No findings & NA & $(2)$ \\
\hline Morita et al, 2009 & L3-L5 & Isointense & Low & + & + & None & & (15) \\
\hline Beitzke et al, 2010 & $\mathrm{~T} 12-\mathrm{S} 1$ & NA & NA & + & + & Increased & NA & (16) \\
\hline Teo et al, 2012 & T11-L4 & NA & Slightly high & NA & + & None & & (17) \\
\hline Cugati et al, 2012 & L2-L3 & Isointense & Isointense & NA & + & None & & (18) \\
\hline Iwasaki et al, 2012 & T12-L1 & NA & Slightly high & + & + & None & & (19) \\
\hline Nishida et al, 2012 & T12-L2 & Low & Slightly high & + & + & Increased & NA & (20) \\
\hline Nakashima et al, 2014 & $\mathrm{~T} 12-\mathrm{S} 1$ & Low & Low & + & + & Increased & NA & (21) \\
\hline Broen et al, 2014 & L2-L5 & NA & Low & + & + & Increased & NA & (22) \\
\hline Broen et al, 2014 & T12-L5 & NA & Low & + & + & None & & (22) \\
\hline Shin et al, 2016 & L3-L5 & NA & Low & + & + & None & & (23) \\
\hline Belcastro et al, 2016 & L2-L4 & NA & NA & + & + & Increased & NA & (24) \\
\hline Wang et al, 2016 & T11-L5 & Low & Isointense & + & + & Increased & 9.6 & $(25)$ \\
\hline Our case & L1-S1 & Low & Low & + & + & Increased & 4.9 & \\
\hline
\end{tabular}

MR, magnetic resonance; PET/CT, positron emission tomography/computed tomography; WI, weighted image; FDG, fluoro-deoxy-glucose; SUV, standardized uptake value; NA, not available; L, lumbar; T, thoracic.

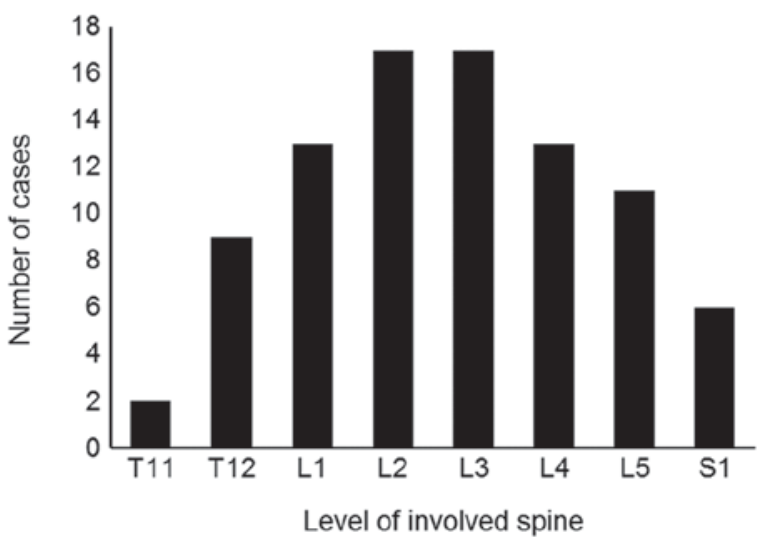

Figure 5. Frequency of involvement of different vertebral levels by cauda equina lymphomas. The levels from L1 to L4 of the spine are frequently involved in lymphoma as seen on MR images. the maximum SUV for CEL was reported in only two cases including our case, the average or cut-off of the maximum SUV for CEL is unclear. On the other hand, the maximum SUV for NL with multiple lesions ranges from 4.9 to 13.0 in multiple peripheral nerves (31). Therefore, in NL including CEL, the SUV of the lesion may not be as high as in general DLBCL. In this case, the reason for the low SUV max of CEL may reflect good prognostic biological characteristics, as this patient with CEL maintained no evidence of disease for 6 years after treatment. The maximum SUV on FDG-PET/CT for newly diagnosed patients with DLBCL is an important predictor of disease progression (30). FDG-PET/CT examination in CEL is also useful for evaluating lesion spread, staging of lymphoma, and response to therapy (25). However, further information on the maximum SUV for NL including CEL is required. 
Treatment for PCNSLs includes radiotherapy alone, chemotherapy alone, and radiation therapy combined with chemotherapy. The reported treatments for primary CEL include three cases treated with radiation alone, seven with chemotherapy alone, and 10 with radiation combined with chemotherapy. The most effective chemotherapeutic regimens for PCNSLs are high-dose methotrexate and multimodal therapy such as adding other chemotherapeutic agents with or without radiation (32). Ferreri et al reported that the addition of high-dose cytarabine to high-dose methotrexate and radiation improves the overall response rate from 40 to $69 \%$ and prolongs progression-free survival from 3 to 18 months (33). In our case, the patient was treated with combination therapy that included high-dose methotrexate and high-dose cytarabine, and he has been living long-term for over 6 years with no evidence of disease. Major toxic effects of chemotherapy with high-dose cytarabine plus methotrexate include neutropenia, thrombocytopenia, and anemia, at a frequency of $90 \%$ or more (32). In our case, the patient showed neutropenia and Grade 3-4 anemia. Survival data for primary CEL, including our case, were available for 21 of the 23 cases. Thirteen patients were alive at the last follow-up observation (follow-up period was 3 to 81 months).

In summary, primary CEL is a rare tumor among NL. This is the first summary of the 23 reported cases, including our present additional case, of primary CEL in terms of the clinical characteristics, laboratory data, analysis of CSF, and features of MR imaging and FDG-PET/CT. Clinical symptoms of CEL are more common in cases of cauda equina syndrome, but are rarely seen in cases of radiculopathy. The typical features seen on MR imaging are enlargement of the cauda equina with iso- or low intensity relative to the spinal cord signal on both T1- and T2-weighted imaging and enhancement of the cauda equina on contrast MR images. Although a few reports of FDG-PET/CT findings are available, FDG accumulation in CEL appears to be increased, but to a lesser extent than in general DLBCL. For the early adjunct diagnosis of CEL, measuring sIL-2R in the CSF, CSF cytology, and immunotyping of CSF are useful, but histopathological analysis following a biopsy of the cauda equina is necessary for definitive diagnosis. The standard treatment for CEL is chemotherapy using high-dose methotrexate, and prognosis is expected to be better when starting treatment early.

\section{Acknowledgements}

The authors would like to thank Dr Hirofumi Konishi, Department of Neurology, University of Toyama (Toyama, Japan) and Dr Jun Murakami, The Third Department of Internal Medicine, University of Toyama for providing specialized discussion on diagnosis and therapy.

\section{Funding}

This study was supported by the Japan Society for the Promotion of Science (JSPS), grant no. JP17K16681.

\section{Availability of data and materials}

All data generated or analyzed during this study are included in this published article.

\section{Authors' contributions}

KS and TY made substantial contributions to conception and design. KS and TH contributed to analysis and interpretation of data. MK and TK assisted in the data analysis. YK was involved in the surgical treatment. TY and YK were involved in drafting the manuscript or revising it critically for important intellectual content. KS made a critical revision of the article for important intellectual content. All authors approved the final version of the manuscript.

\section{Ethics approval and consent to participate}

This report was approved by the Ethics Committee, University of Toyama (Toyama, Japan) and clinical research (no. 21-22) was granted.

\section{Consent for publication}

Written informed consents were obtained from the patient for publication of this report and accompanying images.

\section{Competing interests}

The authors declare that they have no competing interests.

\section{References}

1. Grisariu S, Avni B, Batchelor TT, van den Bent MJ, Bokstein F, Schiff D, Kuittinen O, Chamberlain MC, Roth P, Nemets A, et al: Neurolymphomatosis: An international primary CNS lymphoma collaborative group report. Blood 115: 5005-5011, 2010.

2. Khong P, Pitham T and Owler B: Isolated neurolymphomatosis of the cauda equina and filum terminale: Case report. Spine (Phila Pa 1976) 33: E807-E811, 2008.

3. Baehring JM, Damek D, Martin EC, Betensky RA and Hochberg FH: Neurolymphomatosis. Neuro Oncol 5: 104-115, 2003.

4. Lagarde S, Tabouret E, Matta M, Franques J, Attarian S, Pouget J, Maues De Paula A, Figarella-Branger D, Dory-Lautrec P, Chinot $\mathrm{O}$ and Barrié M: Primary neurolymphomatosis diagnosis and treatment: A retrospective study. J Neurol Sci 342: 178-181, 2014.

5. Deckert M, Paulus W, Kluin PM and Ferry JA: Diffuse large B-cell lymphoma of the CNS. Louis DN, Ohgaki H, Wiestler OD and Cavenee WK (eds). In: WHO Classification of Tumours of the Central Nervous System. WHO/IARC Classification of Tumours, Revised 4th edition. Vol 1. IAR Press, Lyon, pp272-275, 2016.

6. Mauney M and Sciotto CG: Primary malignant lymphoma of the cauda equina. Am J Surg Pathol 7: 185-190, 1983.

7. Toner GC, Holmes R, Sinclair RA, Tang SK and Schwarz MA: Central nervous system lymphoma: Primary lumbar nerve root infiltration. Acta Haematol 81: 44-47, 1989.

8. Klein P, Zientek G, VandenBerg SR and Lothman E: Primary CNS lymphoma: Lymphomatous meningitis presenting as a cauda equina lesion in an AIDS patient. Can J Neurol Sci 17: 329-331, 1990.

9. Knopp EA, Chynn KY and Hughes J: Primary lymphoma of the cauda equina: Myelographic, CT myelographic, and MR appearance. AJNR Am J Neuroradiol 15: 1187-1189, 1994.

10. Ooi GC, Peh WC and Fung CF: Case report: Magnetic resonance imaging of primary lymphoma of the cauda equina. $\mathrm{Br} \mathrm{J}$ Radiol 69: 1057-1060, 1996.

11. Giobbia M, Carniato A, Scotton PG, Vaglia A and Marchiori GC: Primary EBV-associated cauda equina lymphoma. J Neurol 246: 739-740, 1999.

12. Zagami AS and Granot R: Non-Hodgkin's lymphoma involving the cauda equina and ocular cranial nerves: Case reports and literature review. J Clin Neurosci 10: 696-699, 2003. 
13. Kumar N and Dyck PJ: Hypertrophy of the nerve roots of the cauda equina as a paraneoplastic manifestation of lymphoma. Arch Neurol 62: 1776-1777, 2005.

14. Tajima Y, Sudo K and Matumoto A: Malignant lymphoma originating in the cauda equina mimicking the inflammatory polyradiculoneuropathy. Intern Med 46: 1029-1032, 2007.

15. Morita M, Osawa M, Naruse $\mathrm{H}$ and Nakamura $\mathrm{H}$ : Primary NK/T-cell lymphoma of the cauda equina: A case report and literature review. Spine (Phila Pa 1976) 34: E882-E885, 2009.

16. Beitzke M, Enzinger C, Beitzke D, Neureiter D, Ladurner G and Fazekas F: Primary leptomeningeal lymphoma of the cauda equina: A rare cause of radiculopathy. J Neurol 257: 1734-1737, 2010.

17. Teo MK, Mathieson C, Carruthers R, Stewart W and Alakandy L: Cauda equina lymphoma-a rare presentation of primary central nervous system lymphoma: Case report and literature review. $\mathrm{Br}$ J Neurosurg 26: 868-871, 2012.

18. Cugati G, Singh M, Symss NP, Pande A, Vasudevan MC and Ramamurthi R: Primary spinal intradural extramedullary lymphoma causing cauda equina syndrome. J Craniovertebr Junction Spine 3: 58-61, 2012.

19. Iwasaki M, Hida K, Yano S, Aoyama T, Kaneko S and Iwasaki Y: Primary cauda equina lymphoma treated with high-dose methotrexate. Neurol Med Chir (Tokyo) 52: 679-683, 2012.

20. Nishida H, Hori M and Obara K: Primary B-cell lymphoma of the cauda equina, successfully treated with high-dose methotrexate plus high-dose cytarabine: A case report with MRI findings. Neurol Sci 33: 403-407, 2012.

21. Nakashima H, Imagama S, Ito Z, Ando K, Kobayashi K, Ukai J, Muramoto A, Shinjyo R, Matsumoto T, Yamauchi I, et al: Primary cauda equina lymphoma: Case report and literature review. Nagoya J Med Sci 76: 349-354, 2014.

22. Broen M, Draak T, Riedl RG and Weber WE: Diffuse large B-cell lymphoma of the cauda equina. BMJ Case Rep 2014: pii: bcr2014205950, 2014

23. Shin HK, Oh SK, Woo CG, Huh JR, Suh CW and Jeon SR: Cauda equina lymphoma mimicking non-neoplastic hypertrophic neuropathy of the cauda equina: A case report. Br J Neurosurg 30: 678-680, 2016.

24. Belcastro V, Bellcocchi S, Patriarca C, Gini G, Piola M, Barca $S$ and Arnaboldi M: Cauda equina syndrome due to large B-cell lymphoma: A case report. Neurol Sci 37: 825-827, 2016.
25. Wang G, Liu Y and He F: Primary lymphoma involving cranial nerves and cauda equina detected by (18)F-FDG PET/CT and MRI. Nuklearmedizin 55: N46-N48, 2016.

26. Hochberg FH and Miller DC: Primary central nervous system lymphoma. J Neurosurg 68: 835-853, 1988.

27. Goto N, Tsurumi H, Goto H, Shimomura YI, Kasahara S, Hara T, Yasuda I, Shimizu M, Murakami N, Yoshikawa T, et al: Serum soluble interleukin-2 receptor (sIL-2R) level is associated with the outcome of patients with diffuse large B cell lymphoma treated with R-CHOP regimens. Ann Hematol 91: 705-714, 2012.

28. Kitai R, Sasaki H, Matsuda K, Tsunetoshi K, Yamauchi T, Neishi H, Matsumura K, Tsunoda A, Takeuchi H, Sato K and Kikuta K: Measurement and cellular sources of the soluble interleukin-2 receptor in primary central nervous system lymphoma. Brain Tumor Pathol 30: 34-39, 2013.

29. Boukhris S, Magy L, Kabore R, Mabrouk T, Li Y, Sindou P, Tabaraud F and Vallat JM: Atypical electrophysiologic findings in chronic inflammatory demyelinating polyneuropathy (CIDP)-diagnosis confirmed by nerve biopsy. Neurophysiol Clin 34: 71-79, 2004.

30. Miyazaki Y, Nawa Y, Miyagawa M, Kohashi S, Nakase K, Yasukawa $\mathrm{M}$ and Hara M: Maximum standard uptake value of 18F-fluorodeoxyglucose positron emission tomography is a prognostic factor for progression-free survival of newly diagnosed patients with diffuse large B cell lymphoma. Ann Hematol 92: 239-244, 2013.

31. Hong CM, Lee SW, Lee HJ, Song BI, Kim HW, Kang S, Jeong SY, Ahn BC, Lee J and Chae YS: Neurolymphomatosis on F-18 FDG PET/CT and MRI findings: A case report. Nucl Med Mol Imaging 45: 76-78, 2011.

32. Grommes C and DeAngelis LM: Primary CNS lymphoma. J Clin Oncol 35: 2410-2418, 2017.

33. Ferreri AJ, Reni M, Foppoli M, Martelli M, Pangalis GA, Frezzato M, Cabras MG, Fabbri A, Corazzelli G, Ilariucci F, et al: High-dose cytarabine plus high-dose methotrexate versus high-dose methotrexate alone in patients with primary CNS lymphoma: A randomised phase 2 trial. Lancet 374: 1512-1520, 2009. 